\title{
BOUNDS ON THE RUBBLING AND OPTIMAL RUBBLING NUMBERS OF GRAPHS
}

\author{
GYULA Y. KATONA AND NÁNDOR SIEBEN
}

\begin{abstract}
A pebbling move on a graph removes two pebbles at a vertex and adds one pebble at an adjacent vertex. Rubbling is a version of pebbling where an additional move is allowed. In this new move, one pebble each is removed at vertices $v$ and $w$ adjacent to a vertex $u$, and an extra pebble is added at vertex $u$. A vertex is reachable from a pebble distribution if it is possible to move a pebble to that vertex using rubbling moves. The rubbling number is the smallest number $m$ needed to guarantee that any vertex is reachable from any pebble distribution of $m$ pebbles. The optimal rubbling number is the smallest number $m$ needed to guarantee a pebble distribution of $m$ pebbles from which any vertex is reachable. We give bounds for rubbling and optimal rubbling numbers. In particular, we find an upper bound for the rubbling number of $n$-vertex, diameter $d$ graphs, and estimates for the maximum rubbling number of diameter 2 graphs. We also give a sharp upper bound for the optimal rubbling number, and sharp upper and lower bounds in terms of the diameter.
\end{abstract}

\section{INTRODUCTION}

Graph pebbling has its origin in number theory. It is a model for the transportation of resources. Starting with a pebble distribution on the vertices of a simple connected graph, a pebbling move removes two pebbles from a vertex and adds one pebble at an adjacent vertex. We can think of the pebbles as fuel containers. Then the loss of the pebble during a move is the cost of transportation. A vertex is called reachable if a pebble can be moved to that vertex using pebbling moves. There are several questions we can ask about pebbling. How many pebbles will guarantee that every vertex is reachable (pebbling number), or that all vertices are reachable at the same time (cover pebbling number)? How can we place the smallest number of pebbles such that every vertex is reachable (optimal pebbling number)? For a comprehensive list of references for the extensive literature see the survey papers [8, 9].

Graph rubbling is an extension of graph pebbling. In this version, we also allow a move that removes a pebble each from the vertices $v$ and $w$ that are adjacent to a vertex $u$, and adds a pebble at vertex $u$. The basic theory of rubbling and optimal rubbling is developed in [1]. The rubbling number of complete $m$-ary trees are studied in [6], while the rubbling number of caterpillars are determined in [13].

The current paper extends the theory of graph rubbling by providing bounds for the rubbling numbers of graphs. In Section 3, we give an upper bound for the rubbling number in terms of the number of vertices and the diameter of the graph. In Sections $4-5$, we investigate how big the rubbling number of diameter 2 graphs can be. Let $f(n, d)$ be the maximum rubbling number of diameter $d$ graphs with $n$ vertices. We construct a family of graphs whose rubbling numbers match all known values of $f(n, 2)$. We also prove an upper bound for $f(n, 2)$. Similar questions for pebbling are studied in [2, 5], more details are given in Section 5. In Section 6, we give a sharp upper bound for the optimal rubbling number of a graph in terms of the number of vertices. We also give sharp upper and lower bounds in terms of the diameter. Similar results for the optimal pebbling number are presented in [3, 11]. Our results are extensions of these.

2000 Mathematics Subject Classification. 05C99.

Key words and phrases. pebbling, rubbling. 


\section{PReliminaries}

Throughout the paper, let $G$ be a simple connected graph. We use the notation $V(G)$ for the vertex set and $E(G)$ for the edge set. A pebble function on a graph $G$ is a function $p: V(G) \rightarrow \mathbb{Z}$ where $p(v)$ is the number of pebbles placed at $v$. A pebble distribution is a nonnegative pebble function. The size of a pebble distribution $p$ is the total number of pebbles $\sum_{v \in V(G)} p(v)$. We are going to use the notation $p\left(v_{1}, \ldots, v_{n}, *\right)=\left(a_{1}, \ldots, a_{n}, q(*)\right)$ to indicate that $p\left(v_{i}\right)=a_{i}$ for $i \in\{1, \ldots, n\}$ and $p(w)=q(w)$ for all $w \in V(G) \backslash\left\{v_{1}, \ldots, v_{n}\right\}$.

Consider a pebble function $p$ on the graph $G$. If $\{v, u\} \in E(G)$ then the pebbling move $(v, v \rightarrow u)$ removes two pebbles at vertex $v$, and adds one pebble at vertex $u$ to create a new pebble function

$$
p_{(v, v \rightarrow u)}(v, u, *)=(p(v)-2, p(u)+1, p(*)) .
$$

If $\{w, u\} \in E(G)$ and $v \neq w$, then the strict rubbling move $(v, w \rightarrow u)$ removes one pebble each at vertices $v$ and $w$, and adds one pebble at vertex $u$ to create a new pebble function

$$
p_{(v, w \rightarrow u)}(v, w, u, *)=(p(v)-1, p(w)-1, p(u)+1, p(*)) .
$$

A rubbling move is either a pebbling move or a strict rubbling move. A rubbling sequence is a finite sequence $s=\left(s_{1}, \ldots, s_{k}\right)$ of rubbling moves. The pebble function gotten from the pebble function $p$ after applying the moves in $s$ is denoted by $p_{s}$. The pebble function gotten after applying the moves in a multiset $S$ of rubbling moves in any order is denoted by $p_{S}$. The concatenation of the rubbling sequences $r=\left(r_{1}, \ldots, r_{k}\right)$ and $s=\left(s_{1}, \ldots, s_{l}\right)$ is denoted by $r s=\left(r_{1}, \ldots, r_{k}, s_{1}, \ldots, s_{l}\right)$.

A rubbling sequence $s$ is executable from the pebble distribution $p$ if $p_{\left(s_{1}, \ldots, s_{i}\right)}$ is nonnegative for all $i$. A vertex $v$ of $G$ is reachable from the pebble distribution $p$ if there is an executable rubbling sequence $s$ such that $p_{s}(v) \geq 1$. The rubbling number $\rho(G)$ of a graph $G$ is the minimum number $m$ with the property that every vertex of $G$ is reachable from any pebble distribution of size $m$.

The optimal rubbling number $\rho_{\text {opt }}(G)$ of a graph $G$ is the size of a distribution with the least number of pebbles from which every vertex is reachable.

Given a multiset $S$ of rubbling moves on $G$, the transition digraph $T(G, S)$ is a directed multigraph whose vertex set is $V(G)$, and each move $(v, w \rightarrow u)$ in $S$ is represented by two directed edges $(v, u)$ and $(w, u)$. The transition digraph of a rubbling sequence $s=\left(s_{1}, \ldots, s_{n}\right)$ is $T(G, s)=T(G, S)$. where $S=\left\{s_{1}, \ldots, s_{n}\right\}$ is the multiset of moves in $s$. Let $d_{T(G, S)}^{-}$represent the in-degree and $d_{T(G, S)}^{+}$the out-degree in $T(G, S)$. We simply write $d^{-}$and $d^{+}$if the transition digraph is clear from context.

A multiset $S$ of rubbling moves on $G$ is balanced with a pebble distribution $p$ at vertex $v$ if $p_{S}(v) \geq 0$. We say $S$ is balanced with $p$ if $S$ is balanced with $p$ at all $v \in V(G)$, that is, $p_{S} \geq 0$. A multiset of rubbling moves is called acyclic if the corresponding transition digraph has no directed cycles. An element $(v, w \rightarrow u) \in S$ is called an initial move of $S$ if $d^{-}(v)=0=d^{-}(w)$ in the transition digraph.

An important tool is the following result of [1].

Lemma 2.1. (No Cycle) Let $p$ be a pebble distribution on $G$ and $v \in V(G)$. The following are equivalent.

(1) $v$ is reachable from $p$.

(2) There is a multiset $S$ of rubbling moves such that $S$ is balanced with $p$ and $p_{S}(v) \geq 1$.

(3) There is an acyclic multiset $R$ of rubbling moves such that $R$ is balanced with $p$ and $p_{R}(v) \geq 1$.

(4) Vertex $v$ is reachable from $p$ through an acyclic rubbling sequence. 


\section{UPPER BOUND ON THE RUBBLING NUMBER}

All the known upper bounds for the pebbling number $\pi$ are also upper bounds for the rubbling number since $\rho \leq \pi$. The following result is the rubbling version of the upper bound $\pi(G) \leq$ $(n-d)\left(2^{d}-1\right)+1$ [4, Theorem 1]. The difference between the pebbling upper bound and the rubbling upper bound is $2^{d-1}(n-d-1) \geq 0$. The improvement is 0 for $P_{n}$ (the path on $n$ vertices) since then $d=n-1$.

Theorem 3.1. If $G$ is a graph with $n$ vertices and diameter $d$, then

$$
\rho(G) \leq(n-d+1)\left(2^{d-1}-1\right)+2 .
$$

Proof. The statement clearly holds if $n=1$ since then $d=0$, so we may assume that $n \geq 2$. Suppose $p$ is a distribution of pebbles from which vertex $v$ is not reachable. Let $v_{1}$ be a vertex whose distance is maximal from $v$ and let $Q_{1}$ be the shortest path between $v$ and $v_{1}$. Recursively define $v_{i+1}$ to be a vertex in $V(G) \backslash \cup_{j=1}^{i} V\left(Q_{j}\right)$ whose distance is maximal from $v$, and define $Q_{i+1}$ to be the shortest path between $v$ and $v_{i+1}$. The recursion must stop after $m \in \mathbb{N}$ steps. Let $l_{i}$ be the length of $Q_{i}$. Then we have $n>d \geq l_{1} \geq \cdots \geq l_{m} \geq 1$ and $m \leq n-l_{1}$. If

$$
|p| \geq \sum_{i=1}^{m}\left(2^{l_{i}-1}-1\right)+2^{l_{1}-1}+1
$$

then either some $Q_{i}$ has at least $2^{l_{i}}$ pebbles or there are some $Q_{j}$ and $Q_{k}$ with at least $2^{l_{j}-1}$ and $2^{l_{k}-1}$ pebbles respectively. In either case $v$ is reachable. So we must have

$$
\begin{aligned}
|p| & <\sum_{i=1}^{m}\left(2^{l_{i}-1}-1\right)+2^{l_{1}-1}+1 \leq \sum_{i=1}^{m}\left(2^{l_{1}-1}-1\right)+2^{l_{1}-1}+1 \\
& =(m+1)\left(2^{l_{1}-1}-1\right)+2 \leq\left(n-l_{1}+1\right)\left(2^{l_{1}-1}-1\right)+2 \\
& \leq(n-d+1)\left(2^{d-1}-1\right)+2 .
\end{aligned}
$$

The last inequality follows from the fact that $l_{1} \mapsto\left(n-l_{1}+1\right)\left(2^{l_{1}-1}-1\right)$ is increasing for $0<l_{1}<n$.

The upper bound is sharp for $d=0$ since $\rho\left(K_{1}\right)=1$ and for $d=1$ since $\rho\left(K_{n}\right)=2$ for $n>1$. It is also sharp for $d=n-1$ since $\rho\left(P_{n}\right)=2^{n-1}$. If the diameter of $G$ is 2 , then the upper bound becomes $\rho(G) \leq n+1$. This is no surprise since $\rho(G) \leq \pi(G)$ and we know [5] that $\pi(G)$ is either $n$ or $n+1$. However, this upper bound is not sharp.

\section{LOWER BOUND FOR $f(n, 2)$}

There is no lower bound that forces $\rho$ to grow with the number of vertices of the graph. In fact $\rho\left(K_{n}\right)=2$ for all $n$. The only known lower bound $\rho(G) \geq 2^{d}$ for the rubbling number $\rho$ is coming from the diameter $d$ of the graph $G$. So we could ask whether $\{\rho(G) \mid \operatorname{diam}(G)=d\}$ is a finite set for all $d \geq 2$. The family of star shaped graphs constructed in [2] can be used to show that this is not the case for $d \geq 3$. For $d=2$ we need a more elaborate construction.

Our aim is to construct a graph $G_{n}$ for any given $n \geq 3$ with diameter 2 and a high rubbling number. Since the graph is not so easy to describe directly by giving the vertex and edge sets, we first define a simpler graph, then we make modifications on it to reach the final construction.

For a positive integer $s$, let $H_{s}$ be the simple graph defined by

$$
\begin{aligned}
& V\left(H_{s}\right)=\{(i, j) \mid 1 \leq i \leq j \leq s\} \\
& E\left(H_{s}\right)=\left\{\left\{\left(i_{1}, j_{1}\right),\left(i_{2}, j_{2}\right)\right\} \mid i_{1}=i_{2} \text { or } j_{1}=j_{2}\right\} .
\end{aligned}
$$



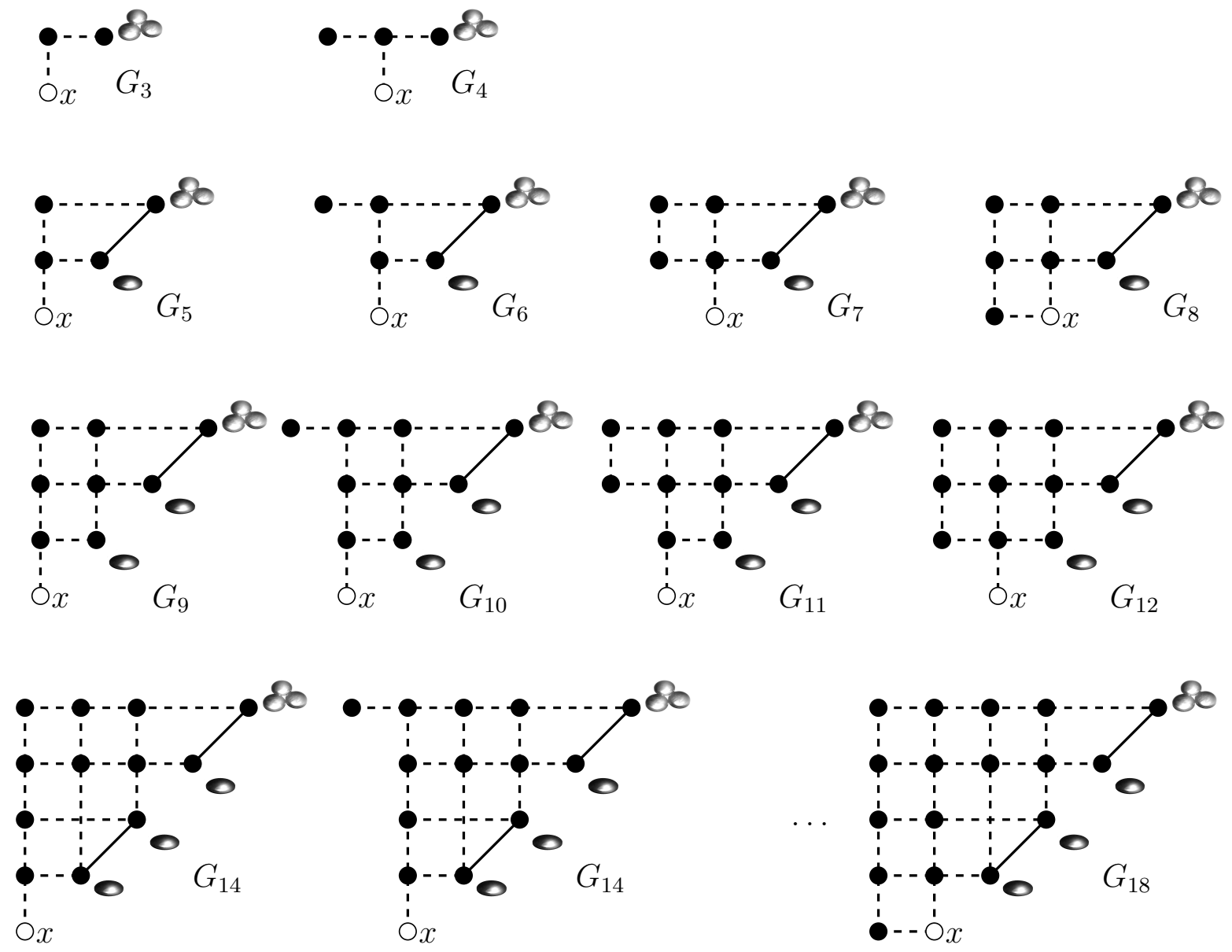

Figure 4.1. Schematic representation of the graphs $G_{3}, \ldots, G_{18}$. The solid lines are edges of the graphs. The dashed lines indicate the fact that any two vertices on a horizontal or a vertical line are connected by an edge. The goal vertex $x$ is not reachable from the pebble distributions shown on the figures.

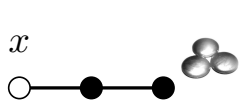

$G_{3}$

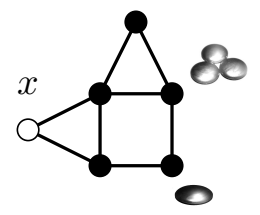

$G_{6}$

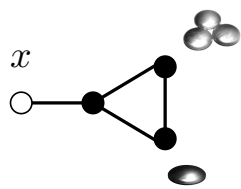

$G_{4}$

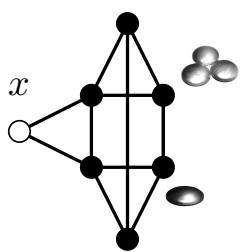

$G_{7}$

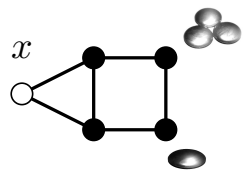

$G_{5}$

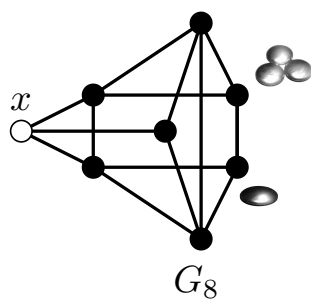

Figure 4.2 . The graphs $G_{3}, \ldots, G_{8}$. The goal vertex $x$ is not reachable from the pebble distributions shown on the figures. 
Clearly $\left|V\left(H_{s}\right)\right|=(s+1) s / 2$. Now we show that $\operatorname{diam}\left(H_{n}\right)=2$. Take two different vertices $\left(i_{1}, j_{1}\right)$ and $\left(i_{2}, j_{2}\right)$ where $i_{1} \leq i_{2}$. If either $i_{1}=i_{2}$ or $j_{1}=j_{2}$, then they are adjacent. Otherwise $\left(i_{1}, j_{2}\right) \in V\left(H_{s}\right)$ is a common neighbor of $\left(i_{1}, j_{1}\right)$ and $\left(i_{2}, j_{2}\right)$, so their distance is 2 .

Now we modify $H_{s}$ by deleting a few vertices and adding a few more edges in the following way. If $s$ is odd and $s \geq 3$, then delete the vertices

$$
(s-1, s),(s-3, s-2),(s-5, s-4), \ldots,(2,
$$

and add the edges

$$
\{(s, s),(s-1, s-1)\},\{(s-2, s-2),(s-3, s-3)\}, \ldots,\{(3,3),(2,2)\} .
$$

If $s$ is even, then delete the vertices

$$
(s-1, s),(s-3, s-2),(s-5, s-4), \ldots,(3,4)
$$

and add the edges

$$
\{(s, s),(s-1, s-1)\},\{(s-2, s-2),(s-3, s-3)\}, \ldots,\{(4,4),(3,3)\} .
$$

Let $H_{s}^{\prime}$ denote the graph that is obtained. Clearly $\left|V\left(H_{s}^{\prime}\right)\right|=(s+1) s / 2-\lfloor(s-1) / 2\rfloor$. One can see that $\operatorname{diam}\left(H_{s}^{\prime}\right)=2$ holds too, since any pair of vertices whose unique common neighbor was deleted, now is either connected by an edge or has a new common neighbor on the spine.

Definition 4.1. If for a given $n \geq 3$ we have $\left|V\left(H_{s}^{\prime}\right)\right|=n$ for some $s$, then let $G_{n}=H_{s}^{\prime}$. Thus, we have the construction of $G_{n}$ for $n=3,5,9,13, \ldots$ For the values of $n$ where $\left|V\left(H_{s}^{\prime}\right)\right|<$ $n<\left|V\left(H_{s+1}^{\prime}\right)\right|$, the construction is given by adding some vertices and edges to $H_{s}^{\prime}$. We add the vertices $(0, s),(0, s-1),(0, s-2), \ldots,(0,1)$ one by one until we reach the required $n$ vertices. A new vertex $(0, j)$ is adjacent to another vertex $\left(i^{\prime}, j^{\prime}\right)$ if either $i^{\prime}=0$ or $j=j^{\prime}$.

A visualization of the graph family $G_{n}$ is shown in Figures 4.1 and 4.2 , Roughly speaking, we add the new vertices on the left of the graph, starting at the top row and continuing towards the bottom. We create new edges to keep the general edge structure of the graph. We stop adding new vertices before we reach the number of vertices in $H_{s+1}^{\prime}$. This means that we stop at vertex $(0,1)$ if $s$ is odd and stop at $(0,2)$ if $s$ is even. Graphs $G_{8}$ and $G_{12}$ shown in Figure 4.1 illustrate these differently placed last new vertices. Note that $G_{9}=H_{4}^{\prime}$ and $G_{13}=H_{5}^{\prime}$.

Note that

$$
\begin{aligned}
\left|V\left(H_{s}^{\prime}\right)\right|+s & =(s+1) s / 2-\lfloor(s-1) / 2\rfloor+s \\
& =(s+2)(s+1) / 2-\lfloor s / 2\rfloor-1=\left|V\left(H_{s+1}^{\prime}\right)\right|-1
\end{aligned}
$$

if $s$ is odd, and $\left|V\left(H_{s}^{\prime}\right)\right|+s-1=\left|V\left(H_{s+1}^{\prime}\right)\right|-1$ if $s$ is even.

The $i$-th row of $G_{n}$ is $R_{i}=\left\{\left(i^{\prime}, j^{\prime}\right) \in V\left(G_{n}\right) \mid i^{\prime}=i\right\}$ while the $i$-th column of $G_{n}$ is $C_{i}=\left\{\left(i^{\prime}, j^{\prime}\right) \in V\left(G_{n}\right) \mid j^{\prime}=j\right\}$. The spine of $G_{n}$ is $\left\{(i, i) \in V\left(G_{n}\right)\right\}$. A short calculation shows that $G_{n}$ has $\lfloor\sqrt{2 n-1}\rfloor$ rows. It is easy to see that $\operatorname{diam}\left(G_{n}\right)=2$.

Proposition 4.2. For $n \geq 3$ we have $\rho\left(G_{n}\right) \leq\lfloor\sqrt{2 n-1}\rfloor+2$.

Proof. Let $k=\lfloor\sqrt{2 n-1}\rfloor$ be the number of rows in $G_{n}$. Let $p$ be a pebble distribution on $G_{n}$ containing $k+2$ pebbles and suppose that a goal vertex $x=\left(i_{0}, j_{0}\right)$ is not reachable. We are going to define some collections of rows and columns, but in one case it is just part of the columns. So let us first define the partial columns: $C_{i}^{\prime}=\left\{\left(i^{\prime}, j^{\prime}\right) \mid i^{\prime}=i\right.$ and $\left.j^{\prime}<j_{0}\right\}$. Now let

$$
\begin{gathered}
\mathcal{R}=\left\{R_{j} \mid j>j_{0}\right\}, \quad R=\cup \mathcal{R}, \\
\mathcal{C}=\left\{C_{i}^{\prime} \mid i \neq i_{0}\right\}, \quad C=\cup \mathcal{C}, \\
X=R_{j_{0}} \cup C_{i_{0}}
\end{gathered}
$$

as shown in Figure 4.3. Then $|\mathcal{R}|=k-j_{0}, j_{0}-2 \leq|\mathcal{C}| \leq j_{0}-1$ (note that $C_{0}^{\prime}$ may be empty) and $R \cup C \cup X=V\left(G_{n}\right)$. 


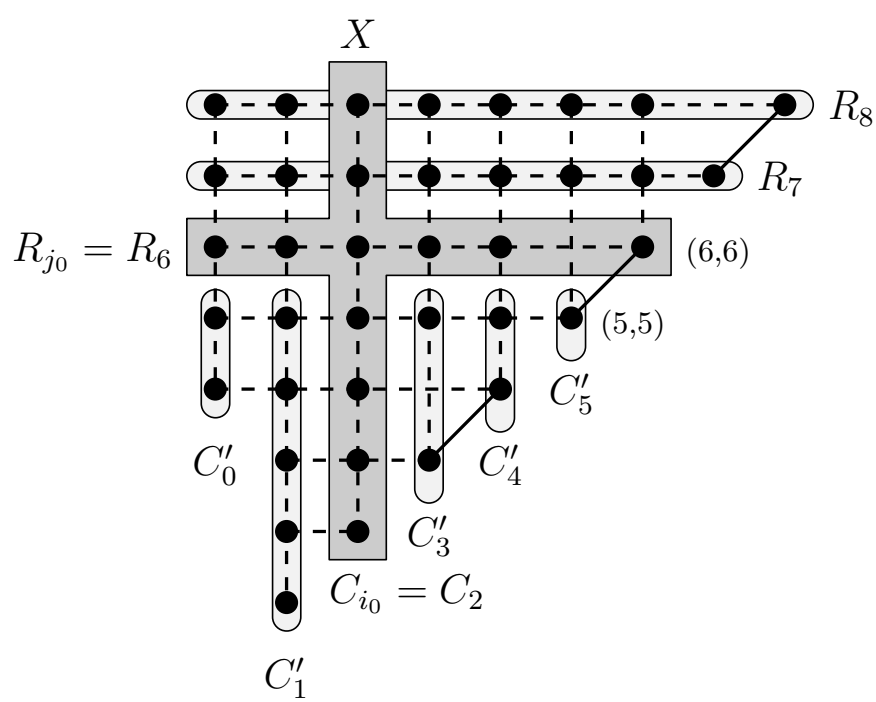

Figure 4.3. The sets $\mathcal{R}, \mathcal{C}$ and $X$..

If $R_{j} \in \mathcal{R}$ and there are two or three pebbles on $R_{j}$, then we can apply a rubbling move on those pebbles and move a pebble to $C_{i_{0}}$. Row $R_{j}$ cannot have 4 or more pebbles, because then we can move two pebbles to $X$, so $x$ would be reachable. Similarly, if $C_{i}^{\prime} \in \mathcal{C}$ and $C_{i}^{\prime}$ has two or three pebbles, then we can move a pebble to $R_{j_{0}}$, while if it has 4 or more pebbles, then we can move two pebbles to $X$. In the exceptional case, when $R_{j_{0}}$ has no vertex in the $i$-th column (like the 5 th column on Figure 4.3), we can move the pebbles along the spine to $\left(i+1, j_{0}\right)$ instead of $\left(i, j_{0}\right)$.

If we can move two pebbles to $X$, then $x$ is clearly reachable. That means that if $R$ or $C$ has one or two more pebbles than the size of $\mathcal{R}$ or $\mathcal{C}$ respectively, then we can move one pebble to $X$. If it has three more pebbles, then we can move two pebbles to $X$. So if either $R$ or $C$ contains enough pebbles to move two pebbles to $X$ or both of them contains enough pebbles to move one pebble to $X$, then $x$ is reachable.

We know that $p$ can have at most one pebble on $X$. If $X$ has no pebbles, then the total number of pebbles cannot be more than

$$
|\mathcal{R}|+|\mathcal{C}|+2 \leq\left(k-j_{0}\right)+\left(j_{0}-1\right)+2=k+1
$$

which is not possible. If $X$ has exactly one pebble, then the total number of pebbles cannot be more than

$$
|\mathcal{R}|+|\mathcal{C}|+1 \leq\left(k-j_{0}\right)+\left(j_{0}-1\right)+1=k
$$

which is not possible either.

In the proof of our next result, we are going to need to keep track of the movement of pebbles during rubbling moves. For this purpose, we need to replace our pebbles with dependency sets.

Definition 4.3. A dependency distribution is a partition $\mathcal{P}$ of an initial pebble set together with a location function $l: \mathcal{P} \rightarrow V(G)$. The elements of $\mathcal{P}$ are called dependency sets or simply pebbles.

We think of a dependency set as a pebble with some additional information about the history of the pebble. Dependency distributions replace pebble distributions. Given a pebble distribution $p$ containing $m$ pebbles, we can create a corresponding dependency distribution $\mathcal{P}=\{\{1\}, \ldots,\{m\}\}$ such that $|\{A \in \mathcal{P} \mid l(A)=v\}|=p(v)$ for all $v \in V(G)$. 
Definition 4.4. If $l(A)$ and $l(B)$ are both adjacent to $u$, then the rubbling move $(A, B \rightarrow u)$ removes the dependency sets $A$ and $B$ from $\mathcal{P}$ at the vertices $l(A)$ and $l(B)$ respectively, and adds a new dependency set $A \cup B$ to $\mathcal{P}$ with location $l(A \cup B)=u$. A vertex $v$ is reachable from a dependency distribution if a dependency set $A$ with $l(A)=v$ can be created using rubbling moves.

Note that the rubbling move $(A, B \rightarrow u)$ is essentially the rubbling move $(l(A), l(B) \rightarrow u)$ with some additional information about the history of the pebbles. It is clear that a vertex is reachable from a pebble distribution if and only if it is reachable from the corresponding dependency distribution. Also note that in a rubbling move $(A, B \rightarrow u)$ we must have $A \cap B=\emptyset$.

Definition 4.5. Let $\left(s_{1}, \ldots, s_{k}\right)$ be a sequence of rubbling moves in a dependency distribution. We say $s_{i}=(A, B \rightarrow u)$ is dependent on $s_{j}=(C, D \rightarrow w)$ if $C \cup D \subseteq A \cup B$. We say that $s_{i}$ and $s_{j}$ are independent if $A \cup B$ and $C \cup D$ are disjoint.

Roughly speaking, $s_{i}$ and $s_{j}$ are independent if they rely on two disjoint sets of pebbles. It is clear that dependence of rubbling moves is a transitive relation. Also note that $s_{i}$ and $s_{j}$ are independent if and only if neither $s_{i}$ is dependent on $s_{j}$ nor $s_{j}$ is dependent on $s_{i}$.

Example 4.6. Consider the initial dependency distribution $l(\{1\},\{2\},\{3\},\{4\},\{5\})=$ $(w, w, w, w, v)$ and the sequence of rubbling moves

$$
s_{1}=(\{1\},\{2\} \rightarrow u), s_{2}=(\{3\},\{4\} \rightarrow v), s_{3}=(\{3,4\},\{5\} \rightarrow u), s_{4}=(\{1,2\},\{3,4,5\} \rightarrow x) .
$$

Then $s_{1}$ and $s_{3}$ are independent but $s_{4}$ depends on $s_{1}$.

Proposition 4.7. For $n \geq 3$ we have $\rho\left(G_{n}\right)>\lfloor\sqrt{2 n-1}\rfloor+1$.

Proof. Let $k=\lfloor\sqrt{2 n-1}\rfloor$ be the largest column index in $G_{n}$. We show that the goal vertex $x:=(1,1)$ is not reachable from the pebble distribution that has a single pebble on vertex $(i, i)$ for $1<i<k$ and three pebbles on vertex $(k, k)$ as shown in Figures 4.1 and 4.2 . To see this, we show that $x$ is not reachable from the dependency distribution $\mathcal{P}=\{\{1\}, \ldots,\{k+1\}\}$ with

$$
l(\{1\}, \ldots,\{k-2\},\{k-1\},\{k\},\{k+1\})=((2,2), \ldots,(k-1, k-1),(k, k),(k, k),(k, k)) .
$$

For a contradiction suppose that $x$ is reachable from $\mathcal{P}$, that is, there is a sequence $s_{1}, \ldots, s_{m}$ of rubbling moves that creates a dependency set at $x$.

Let us call a rubbling move $(A, B \rightarrow u)$ horizontal if $l(A), l(B)$ and $u$ are all contained in the same row of $G_{n}$. To reach the goal vertex $x$, we must use a rubbling move involving vertices on $C_{1} \cup C_{0}$. There are no pebbles on these vertices originally and the only way to move a new pebble there is to use a horizontal rubbling move. So there must be at least two independent horizontal moves $s_{i}=(A, B \rightarrow u)$ and $s_{j}=(C, D \rightarrow w)$ in our rubbling sequence. We show that this is not possible.

Since $s_{i}$ and $s_{j}$ are independent, at least one of the sets $A \cup B$ and $C \cup D$ contains at most one element of $\{k-1, k, k+1\}$. Roughly speaking, this means that both $s_{i}$ and $s_{j}$ cannot rely on more than one pebble available at vertex $(k, k)$ in $\mathcal{P}$ since there are only three pebbles there. So we can assume that $(A \cup B) \cap\{k, k+1\}=\emptyset$.

Now we create a new dependency distribution $\tilde{\mathcal{P}}=\mathcal{P} \backslash\{\{k\},\{k+1\}\}$ by removing the two pebbles from $\mathcal{P}$ that $s_{i}$ does not rely on for sure. We also remove the rubbling moves from $\left(s_{1}, \ldots, s_{m}\right)$ that are dependent on $k$ or $k+1$. More precisely, we remove the rubbling moves of the form $(K, L \rightarrow v)$ for which $(K \cup L) \cap\{k, k+1\} \neq \emptyset$. The resulting rubbling sequence $\left(\tilde{s}_{1}, \ldots, \tilde{s}_{\tilde{m}}\right)$ is executable from $\tilde{\mathcal{P}}$ and contains $s_{i}$.

Let us call $L_{i}=R_{i} \cup C_{i}$ for $i \geq 1$ a line of $G_{n}$. Line $L_{i}$ contains the spine vertex $(i, i)$. Note that all vertices on the spine are contained in exactly one line, and all other vertices are contained in at most two lines. We say that a pebble configuration is forbidden if there is a line 


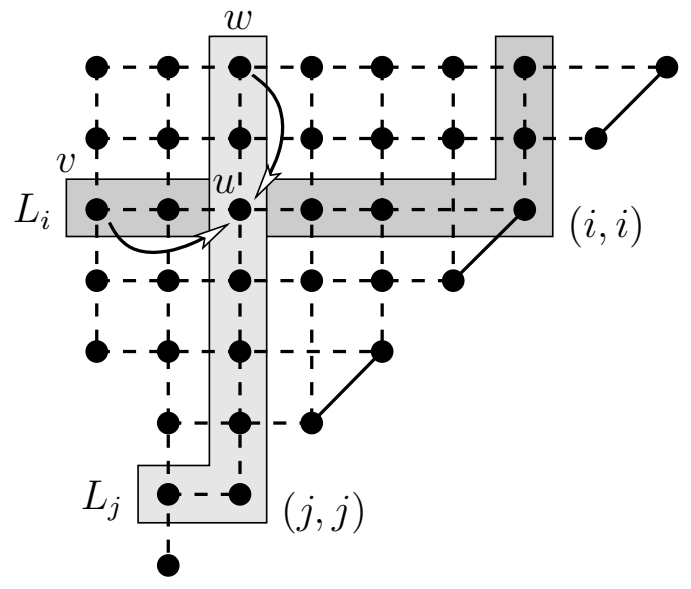

(a)

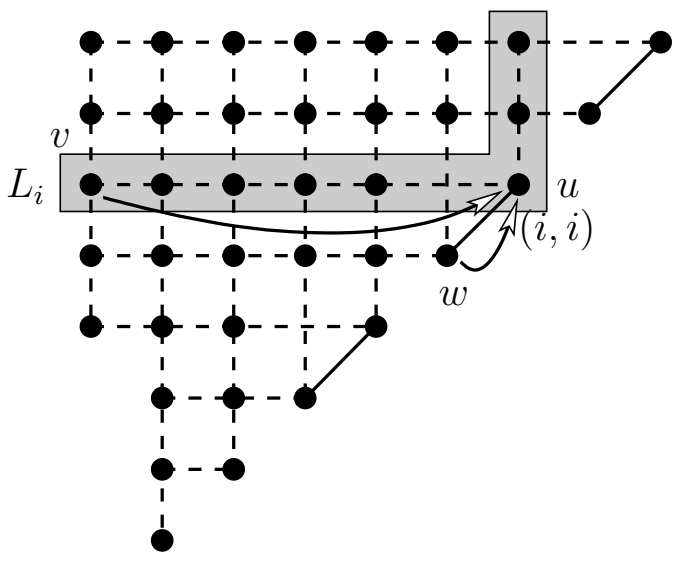

(b)

Figure 4.4. Possible ways to move a pebble to the line $L_{i}$.

\begin{tabular}{|c|c|c|c|c|c|c|c|c|}
\hline$n$ & 3 & 4 & 5 & 6 & 7 & 8 & 9 & 10 \\
\hline$f(n, 2)$ & 4 & 4 & 5 & 5 & 5 & 5 & 6 & $?$ \\
\hline$\rho\left(G_{n}\right)$ & 4 & 4 & 5 & 5 & 5 & 5 & 6 & 6 \\
\hline
\end{tabular}

TABLE 1. Rubbling numbers of $G_{n}$ and all known maximum rubbling numbers for diameter 2 graphs with $n$ vertices.

with more than one pebble. Note that two pebbles on a row $R_{i}$ or on a column $C_{i}$ with $i \geq 1$ is a forbidden configuration. It is clear that $\tilde{\mathcal{P}}$ is not a forbidden pebble configurations.

We are going to show that a rubbling move cannot create a forbidden configuration if there was no forbidden configuration before the rubbling move. Suppose that a rubbling move $(A, B \rightarrow u)$ creates a pebble on vertex $u$ of line $L_{i}$. If $u$ is not on the spine, then it is contained in another line $L_{j}$, so all of its neighbors are in $L_{i} \cup L_{j}$. Both $l(A)$ and $l(B)$ cannot be on the same line since there was no forbidden configuration before this step. Thus one of $l(A)$ and $l(B)$ must be on $L_{i}$ while the other must be outside of $L_{i}$ as shown on Figure 4.4(a). If $u$ is on the spine, then it has a neighbor which is on the spine, too. Again, both of $l(A)$ and $l(B)$ cannot be on this vertex, because that is a forbidden configuration. Therefore one of $l(A)$ and $l(B)$ must be on $L_{i}$ again as shown on Figure 4.4(b) since in this case all other neighbors of $u$ are in $L_{i}$.

Thus we can assume that $l(A)=v$ is in $L_{i}$. Since $v$ is in $L_{i}$, we cannot have any other pebble on $L_{i}$ before the rubbling move. So $u$ is the only pebble on $L_{i}$ after the rubbling move and so the rubbling move did not create any forbidden configurations.

We saw that $\left(\tilde{s}_{1}, \ldots, \tilde{s}_{\tilde{m}}\right)$ has the horizontal move $s_{i}$. A horizontal move is only possible if there are two pebbles on a row which is a forbidden configuration. This is a contradiction since we do not have any forbidden configurations during the execution of $\left(\tilde{s}_{1}, \ldots, \tilde{s}_{\tilde{m}}\right)$.

Corollary 4.8. For $n \geq 3$ we have $\rho\left(G_{n}\right)=\lfloor\sqrt{2 n-1}\rfloor+2$.

\section{UPPER BOUND FOR $f(n, 2)$}

Table 1 shows the maximum rubbling numbers

$$
f(n, 2)=\max \{\rho(G)|n=| V(G) \mid \text { and } 2=\operatorname{diam}(G)\}
$$




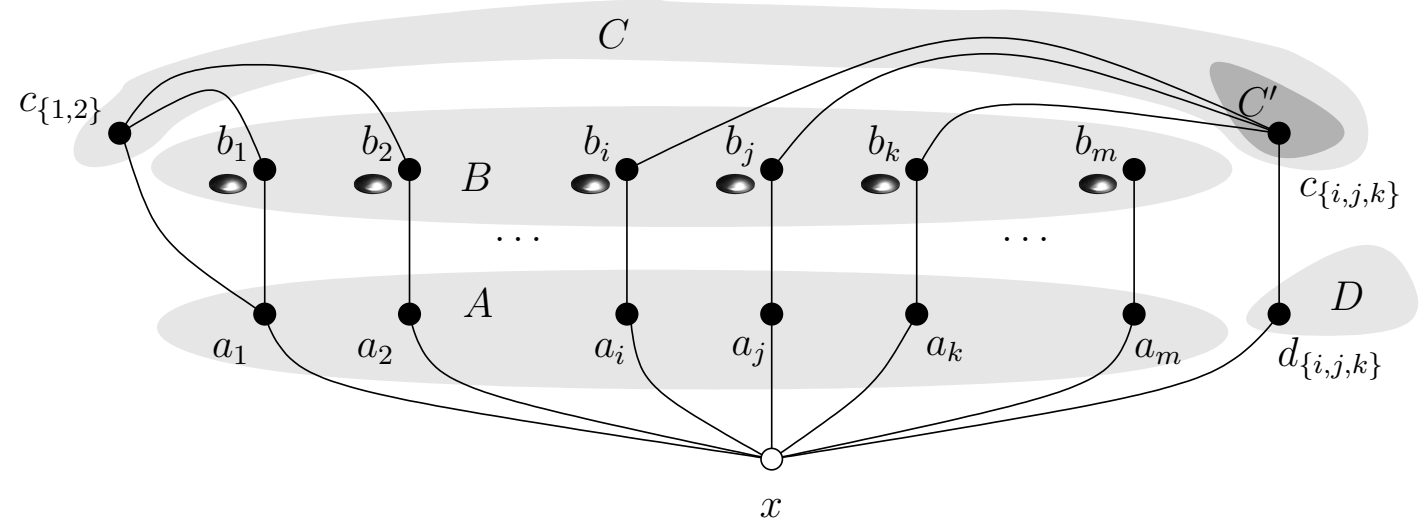

Figure 5.1. The sets $A, B, C, C^{\prime}$ and $D$ in the proof of Proposition 5.3 .

of diameter 2 graphs with $n$ vertices. The values were calculated by a computer program [14]. The program checked all diameter 2 graphs with a given number of vertices. These graphs were generated by Nauty [10]. We have $f(n, 2)=\rho\left(G_{n}\right)$ for $n \in\{3, \ldots, 9\}$. It is not clear whether this is true for all $n$.

Problem 5.1. Is it true that $f(n, 2)=\rho\left(G_{n}\right)$ for all $n \geq 3$ ?

There are more existing results for similar questions about pebbling. It is known [12 that $f(n, 2)=n+1$ since the pebbling number of a diameter 2 graph is either $n$ or $n+1$. A classification of diameter 2 graphs with pebbling number $n+1$ is also known from [5]. Diameter 3 graphs are also studied. In [2], it is shown that $f(n, 3)=\frac{3}{2} n+O(1)$.

The proof of the following result uses the method of [7].

Lemma 5.2. Let $\mathcal{H}$ be a 3-uniform hypergraph on $q$ vertices. If $|E \cap F| \neq 1$ for all $E, F \in \mathcal{H}$, then $|\mathcal{H}| \leq q$.

Proof. Let $\mathbf{v}_{1}, \ldots, \mathbf{v}_{n}$ denote the characteristic vectors of the sets in $\mathcal{H}$. We claim that the characteristic vectors are linearly independent over $G F(2)$. This clearly implies the result.

Since every set contains 3 elements, we have $\mathbf{v}_{i}^{2}=1$ for all $i$ since $3 \equiv_{2} 1$. On the other hand, if $i \neq j$ then $\mathbf{v}_{i} \cdot \mathbf{v}_{j}=0$ since the product is the cardinality of the intersection of the two corresponding sets. If $\sum_{i}^{n} c_{i} \mathbf{v}_{i}=\mathbf{0}$ then multiplying by $\mathbf{v}_{j}$ we obtain $c_{j}=c_{j} \mathbf{v}_{j}^{2}=\mathbf{0} \cdot \mathbf{v}_{j}=0$. This proves our claim.

The set of vertices adjacent to a given vertex $v$ of a graph is denoted by $N(v)$.

Proposition 5.3. Let $G$ be a diameter two graph with goal vertex $x$. Let $p$ be a pebble distribution on $G$ containing $m$ pebbles. If $\{x\} \cup N(x)$ is not reachable using only five pebbles of $p$, then $G$ has at least $\left\lfloor\frac{m^{2}+3}{2}\right\rfloor$ vertices.

Proof. For $v \in N(x)$ let $R(v)=N(v) \cup\{v\} \backslash\{x\}$. Since the diameter of $G$ is two, we must have

$$
V(G) \backslash\{x\}=\bigcup_{v \in N(x)} R(v) .
$$

Since $\{x\} \cup N(x)$ is not reachable using only five pebbles, no $R(v)$ can contain more than two pebbles otherwise we could move a pebble to $v \in N(x)$. So there is a maximal subset $A=\left\{a_{1}, \ldots, a_{m}\right\}$ of $N(x)$ such that $R\left(a_{i}\right)$ contains exactly one pebble on a vertex $b_{i} \in R\left(a_{i}\right)$ for all $i$. Define $B=\left\{b_{1}, \ldots, b_{m}\right\}$ and note that $A$ and $B$ are clearly disjoint. To simplify notation we write $R_{i}$ for $R\left(a_{i}\right)$. Figure 5.1 shows a visualization of these sets. 
If two vertices $b_{i}$ and $b_{j}$ of $B$ are not adjacent, then the diameter condition implies that we can pick a common neighbor $c_{\{i, j\}}$ of $b_{i}$ and $b_{j}$. Note that $c_{\{i, j\}}=c_{\{j, i\}}$. If $i, j, k$ and $l$ are all different, then $c_{\{i, j\}} \neq c_{\{k, l\}}$, otherwise $\left(b_{i}, b_{j} \rightarrow c_{\{i, j\}}\right)\left(b_{k}, b_{l} \rightarrow c_{\{i, j\}}\right)$ would move two pebbles to $c_{\{i, j\}}$ from which $N(x)$ is reachable using only four pebbles.

If $c_{\{i, j\}}=c_{\{i, k\}}$ then we write $c_{\{i, j, k\}}$ for $c_{\{i, j\}}=c_{\{i, k\}}$. Define

$$
\mathcal{J}=\left\{\{i, j\} \mid\left\{b_{i}, b_{j}\right\} \in E(\bar{G})\right\}, \quad C=\left\{c_{\{i, j\}} \mid\{i, j\} \in \mathcal{J}\right\} .
$$

A vertex $b_{i}$ cannot be adjacent to two different vertices $b_{j}$ and $b_{k}$ of $B$, otherwise $\left(b_{j}, b_{k} \rightarrow b_{i}\right)$ would create two pebbles on $b_{i}$, making $a_{i} \in N(x)$ reachable using only three pebbles. Hence the number of edges between the elements of $B$ is at most $\left\lfloor\frac{m}{2}\right\rfloor$ and so $|\mathcal{J}| \geq\left(\begin{array}{c}m \\ 2\end{array}\right)-\left\lfloor\frac{m}{2}\right\rfloor$.

We also introduce a subset $C^{\prime}$ of $C$ by letting

$$
\mathcal{K}=\left\{\{i, j, k\} \mid c_{\{i, j\}}=c_{\{i, k\}}\right\}, \quad C^{\prime}=\left\{c_{\{i, j, k\}} \mid\{i, j, k\} \in \mathcal{K}\right\} .
$$

Though it may happen that $c_{\{i, j\}}=c_{\{i, k\}}=c_{\{j, k\}}$, we have $\left|C \backslash C^{\prime}\right| \geq \mathcal{J}-3\left|C^{\prime}\right|$. Note that $c_{\{i, j\}} \in$ $C \backslash C^{\prime}$ and $\{i, j\} \neq\{k, l\}$ implies $c_{\{i, j\}} \neq c_{\{k, l\}}$. If $c_{\{i, j, k\}}=c_{\left\{i^{\prime}, j^{\prime}, k^{\prime}\right\}}$ then $\{i, j, k\}=\left\{i^{\prime}, j^{\prime}, k^{\prime}\right\}$ otherwise we could move two pebbles to $c_{\{i, j, k\}}$ using only four pebbles. Hence $|\mathcal{K}|=\left|C^{\prime}\right|$.

We can clearly move a pebble to any element of $C$ and so $C \cap A \subseteq C \cap N(x)=\emptyset$. If $k \notin\{i, j\}$ then $c_{\{i, j\}} \notin R_{k}$ otherwise $\left(b_{i}, b_{j} \rightarrow c_{\{i, j\}}\right)\left(b_{k}, c_{\{i, j\}} \rightarrow a_{k}\right)$ would move a pebble to $a_{k} \in N(x)$ using only three pebbles. In particular, $c_{\{i, j\}} \neq b_{k}$ and so $C \cap B=\emptyset$.

Suppose that $c_{\{i, j, k\}} \in C^{\prime}$. Then $c_{\{i, j, k\}} \notin R_{l}$ for $l \notin\{i, j, k\}$ since $c_{\{i, j, k\}}$ is in $\left\{c_{\{i, j\}}, c_{\{i, k\}}, c_{\{j, k\}}\right\}$. We also have $c_{\{i, j, k\}} \notin R_{i}$, otherwise $\left(b_{j}, b_{k} \rightarrow c_{\{i, j, k\}}\right)\left(b_{i}, c_{\{i, j, k\}} \rightarrow a_{i}\right)$ would move a pebble to $a_{i} \in N(x)$ using only three pebbles. Similar arguments show that $c_{\{i, j, k\}} \notin$ $R_{j} \cup R_{k}$. Hence

$$
c_{\{i, j, k\}} \in R\left(d_{\{i, j, k\}}\right) \backslash \bigcup_{l=1}^{m} R_{l} \quad \text { for some } \quad d_{\{i, j, k\}} \in N(x) \backslash A .
$$

Let $D=\left\{d_{\{i, j, k\}} \mid\{i, j, k\} \in \mathcal{K}\right\}$. Then $D$ is disjoint from $A \cup B$ by definition. We also have $D \cap C \subseteq N(x) \cap C=\emptyset$. If $d_{\{i, j, k\}}=d_{\left\{i^{\prime}, j^{\prime}, k^{\prime}\right\}}$ then $\{i, j, k\}=\left\{i^{\prime}, j^{\prime}, k^{\prime}\right\}$, otherwise $\left\{b_{i}, b_{j}, b_{k}, b_{i^{\prime}}, b_{j^{\prime}}, b_{k^{\prime}}\right\}$ would have at least four vertices with pebbles so we could move a pebble to $d_{\{i, j, k\}} \in N(x)$ using only these four pebbles. So $D$ and $C^{\prime}$ has the same number of elements.

The intersection of two elements $\{i, j, k\}$ and $\left\{i, j^{\prime}, k^{\prime}\right\}$ of $\mathcal{K}$ cannot be a singleton $\operatorname{set}\{i\}$, otherwise

$$
\left(b_{j}, b_{k} \rightarrow c_{\{j, k\}}\right)\left(b_{j^{\prime}}, b_{k^{\prime}} \rightarrow c_{\left\{j^{\prime}, k^{\prime}\right\}}\right)\left(c_{\{j, k\}}, c_{\left\{j^{\prime}, k^{\prime}\right\}} \rightarrow b_{i}\right)
$$

would move two pebbles to $b_{i}$ from where $\{x\} \cup N(x)$ would be reachable using only five pebbles. Hence the 3-uniform hypergraph $\mathcal{K}$ satisfies the conditions of Lemma 5.2 and so $|\mathcal{K}| \leq m$.

The result now follows from the calculation

$$
\begin{aligned}
\left|V_{G}\right| & \geq|\{x\}|+|A|+|B|+|C|+|D|=1+m+m+\left|C \backslash C^{\prime}\right|+\left|C^{\prime}\right|+|D| \\
& \geq 1+2 m+|\mathcal{J}|-3\left|C^{\prime}\right|+\left|C^{\prime}\right|+\left|C^{\prime}\right| \geq 1+2 m+\left(\begin{array}{c}
m \\
2
\end{array}\right)-\left\lfloor\frac{m}{2}\right\rfloor-\left|C^{\prime}\right| \\
& =\frac{2+3 m+m^{2}}{2}-\left\lfloor\frac{m}{2}\right\rfloor-|\mathcal{K}| \geq \frac{2+3 m+m^{2}}{2}-\left\lfloor\frac{m}{2}\right\rfloor-m=\left\lfloor\frac{m^{2}+3}{2}\right\rfloor .
\end{aligned}
$$

Proposition 5.4. Let $G$ be a diameter two graph with goal vertex $x$. Let $p$ be a pebble distribution on $G$ containing $m \geq 5$ pebbles. If $x$ is not reachable from $p$ then $G$ has at least $\left\lfloor\frac{(m-5)^{2}+3}{2}\right\rfloor$ vertices. 
Proof. If $p$ satisfies the conditions of Proposition 5.3, then $G$ must have at least $\left\lfloor\frac{m^{2}+3}{2}\right\rfloor$ vertices.

Otherwise there are five pebbles of $p$ from where $\{x\} \cup N(x)$ is reachable. We can remove these pebbles to create a new pebble distribution $q$ containing $m-5$ pebbles. Then $q$ must satisfy the conditions of Proposition 5.3, otherwise we could move two pebbles to $\{x\} \cup N(x)$ and so $x$ would be reachable. So $G$ must have at least $\left\lfloor\frac{(m-5)^{2}+3}{2}\right\rfloor$ vertices.

Proposition 5.5. The rubbling number of a diameter 2 graph with $n$ vertices cannot be larger than $\sqrt{2 n-1}+5$.

Proof. From Proposition 5.4, we know that if we have $m \geq 5$ pebbles on a diameter 2 graph with $n$ vertices one of which is not reachable, then $n \geq\left\lfloor\frac{(m-5)^{2}+3}{2}\right\rfloor$. The contrapositive gives that if a graph has $n<\left\lfloor\frac{(m-5)^{2}+3}{2}\right\rfloor$ vertices and $m \geq 5$, then any goal vertex is reachable from any placement of $m$ pebbles and so the rubbling number is larger than $m$. The result now follows since we have

$$
\begin{aligned}
n<\left\lfloor\frac{(m-5)^{2}+3}{2}\right\rfloor & \Longleftrightarrow n+1 \leq \frac{(m-5)^{2}+3}{2} \\
& \Longleftrightarrow \sqrt{2 n-1}+5 \leq m .
\end{aligned}
$$

Corollary 5.6. We have $\lfloor\sqrt{2 n-1}\rfloor+2 \leq f(n, 2) \leq \sqrt{2 n-1}+5$.

\section{Bounds on the optimal RUBbling NUMBER}

We saw in [1] that $\rho_{\text {opt }}\left(P_{n}\right)=\left\lceil\frac{n+1}{2}\right\rceil$. We show that the path requires the most pebbles for optimal rubbling amongst the graphs with a given number of vertices. The proof follows the ideas of [3].

Proposition 6.1. If $G$ is a tree with $n$ vertices, then $\rho_{\text {opt }}(G) \leq\left\lceil\frac{n+1}{2}\right\rceil$.

Proof. We use induction on $n$. The statement is clearly true for $n \in\{1,2\}$. In the inductive step let $n \geq 3$ and let $v_{1}, v_{2}, \ldots, v_{k}$ be the consecutive vertices of a longest path of $G$. Note that $k \geq 3$. We are going to find a subtree $H$ of $G$ with $n-2$ vertices as shown in Figure 6.1, Then there is a pebble distribution $q$ on $H$ with size $\left\lceil\frac{n-2+1}{2}\right\rceil$ from which every vertex of $H$ is reachable.

If $d\left(v_{2}\right)=2$ then let $H$ be the subtree of $G$ gotten by deleting $v_{1}$ and $v_{2}$. Then every vertex of $G$ is reachable from the pebble distribution $p\left(v_{1}, v_{2}, *\right):=(1,0, q(*))$.

If $d\left(v_{2}\right)>2$ then let $w$ be a vertex that is adjacent to $v_{2}$ but different from $v_{1}$ and $v_{3}$. By the maximality of the chosen path, $w$ must be a leaf vertex. Let $H$ be the subtree of $G$ gotten by deleting $v_{1}$ and $w$. Then every vertex of $G$ is reachable from the pebble distribution $p\left(v_{1}, v_{2}, w, *\right):=\left(0, q\left(v_{2}\right)+1,0, q(*)\right)$.

The size of $p$ is $\left\lceil\frac{n-1}{2}\right\rceil+1=\left\lceil\frac{n+1}{2}\right\rceil$ in both cases as desired.

Corollary 6.2. If $G$ is a connected graph with $n$ vertices, then $\rho_{\text {opt }}(G) \leq\left\lceil\frac{n+1}{2}\right\rceil$.

Proof. Let $H$ be a spanning tree of $G$. Then $\rho_{\text {opt }}(G) \leq \rho_{\text {opt }}(H) \leq\left\lceil\frac{n+1}{2}\right\rceil$ since additional edges cannot increase the optimal rubbling number of a graph.

Since $\rho_{\text {opt }}\left(K_{n}\right)=2$ for $n \geq 2$, there is no useful lower bound for the optimal rubbling number in terms of the number of vertices. We can still find bounds in terms of the diameter. Similar results are presented in [11] for the optimal pebbling number.

Proposition 6.3. If $G$ is a connected graph with diameter $d$, then $\left\lceil\frac{d+2}{2}\right\rceil \leq \rho_{\text {opt }}(G)$. 

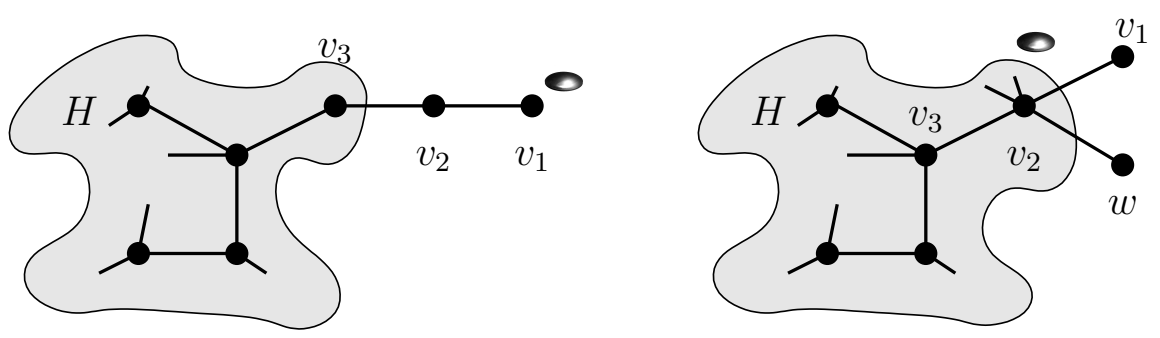

Figure 6.1. The construction of $H$ in the proof of Proposition 6.1.

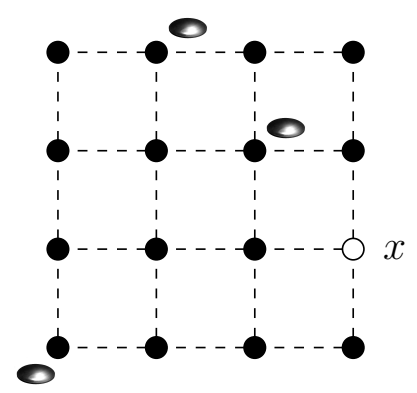

FiguRE 6.2. Schematic representation of a graph with diameter 2. The dashed lines indicate the fact that any two vertices on a dashed line are connected by an edge. The goal vertex $x$ is not reachable from the pebble distribution shown on the figure.

Proof. Let $v_{0}, v_{1}, v_{2}, \ldots, v_{d}$ be a path where this is a shortest path between $v_{0}$ and $v_{d}$. Build a Breadth First Search tree from $v_{0}$. This defines a partition of the vertex set of $G$ into levels: denote the set of vertices at distance $i$ from $v_{0}$ by $L_{i}$. It is clear that $v_{i} \in L_{i}$ holds for $i \in$ $\{0, \ldots, d\}$. Let $P_{d+1}$ denote the graph that is a path of length $d$, and let $V\left(P_{d+1}\right)=\left\{u_{0}, \ldots, u_{d}\right\}$. Define a mapping $\phi: V(G) \rightarrow V\left(P_{d+1}\right)$ such that $\phi(w)=u_{i}$ for all $w \in L_{i}$.

We claim that if a vertex $x$ in $G$ is reachable from a pebble distribution $p$ using a sequence $s$ of rubbling moves, then $\phi(x)$ is reachable in $P_{d+1}$ from the pebble distribution that has $\sum_{w \in L_{i}} p(w)$ pebbles on $u_{i}$.

Suppose that $(a, b \rightarrow c)$ is a rubbling move in $s$. That means that $\{a, c\}$ and $\{b, c\}$ are both edges of $G$. The properties of the BFS tree implies that $a$ and $c$ (also $b$ and $c$ ) are either in the same level or in two neighboring levels. If $a$ and $c$ (or $b$ and $c$ ) are in the same level, then we can delete this rubbling move from the sequence, since $\phi(a)=\phi(c)$ (or $\phi(b)=\phi(c))$, so the pebble which is moved to $c$ by $(a, b \rightarrow c)$ is already on $\phi(c)$ in the corresponding rubbling sequence in $P_{d+1}$.

If $c$ is not in the same level with $a$ and with $b$, then use the rubbling move $(\phi(a), \phi(b) \rightarrow \phi(c))$. It is easy to show by induction that this new rubbling sequence will move a pebble to $\phi(x)$.

The result now follows from this, since $\left\lceil\frac{d+2}{2}\right\rceil=\rho_{\text {opt }}\left(P_{d+1}\right) \leq \rho_{\text {opt }}(G)$.

If the diameter of $G$ is $d$, then every vertex is reachable from the distribution that has $2^{d}$ pebbles on a single vertex. Hence $\rho_{\text {opt }}(G) \leq 2^{d}$. The following example shows that this inequality is sharp.

Proposition 6.4. For all nonnegative integer $d$ there is a graph $G$ with diameter $d$ and $\rho_{\text {opt }}(G)=$ $2^{d}$.

Proof. Let the vertex set of $G$ be the set of points in $\mathbb{N}^{d}$ with coordinates in $\left\{1, \ldots, 2^{d}\right\}$. Let two vertices be connected by an edge if they share all but one of their coordinates. It is clear that 
the diameter of $G$ is $d$. Moreover, any two vertices that has no common coordinate has distance $d$.

Consider a pebble distribution $p$ containing $2^{d}-1$ pebbles. Since there are more possible values at the $i$-th coordinate than the number of pebbles, there must be an $x_{i} \in\left\{1, \ldots, 2^{d}\right\}$ for all $i \in\{1, \ldots, d\}$ such that no pebble has $x_{i}$ as its $i$-th coordinate. Hence the vertex $x=$ $\left\{x_{1}, x_{2}, \ldots, x_{d}\right\}$ does not share any coordinate with any of the vertices containing pebbles. In this way every pebble is at distance $d$ from $x$. Figure 6.2 shows one such pebble distribution on $G$ with vertex $x$ for $d=2$.

Assign a weight $w_{x}(p)$ to the pebble distribution $p$ in the following way: Each pebble in $p$ gets a $1 / 2^{i}$ weight if its distance from $x$ is $i$. Then $w_{x}(p)$ is the sum of the weights for all pebbles in the distribution. Since the initial distribution has $2^{d}-1$ pebbles, each at distance $d$ from $x$, its weight is less than 1 . It is easy to see, that a rubbling move cannot increase the weight. If it removes two pebbles that are at distance $i+1$ and puts a pebble to a vertex which is one closer to $x$, at distance $i$, then the total weight remains the same, but in all other cases it decreases. This shows that any sequence of rubbling moves keeps the weight smaller than 1 . However, if $x$ has a pebble, then the weight is clearly at least 1 . Therefore $x$ is not reachable, so $p$ is not solvable.

Note that our example also serves as an example for a diameter $d$ graph with maximum pebbling number since $2^{d}=\rho_{\text {opt }}(G) \leq \pi_{\text {opt }}(G) \leq 2^{d}$. In fact, our example is essentially a larger version of the example presented in [11, Theorem 2.3]. The larger size allows for a simple proof that also fills a gap in the proof of the original example.

\section{REFERENCES}

1. Christopher Belford and Nándor Sieben, Rubbling and optimal rubbling of graphs., Discrete Math. 309 (2009), no. 10, 3436-3446.

2. Boris Bukh, Maximum pebbling number of graphs of diameter three, J. Graph Theory 52 (2006), no. 4, 353-357.

3. David P. Bunde, Erin W. Chambers, Daniel Cranston, Kevin Milans, and Douglas B. West, Pebbling and optimal pebbling in graphs, J. Graph Theory 57 (2008), no. 3, 215-238.

4. Melody Chan and Anant P. Godbole, Improved pebbling bounds, Discrete Math. 308 (2008), no. 11, $2301-2306$.

5. T. A. Clarke, R. A. Hochberg, and G. H. Hurlbert, Pebbling in diameter two graphs and products of paths, J. Graph Theory 25 (1997), no. 2, 119-128.

6. Lisa Danz, Optimal t-rubbling of complete m-ary trees, REU project report, University of Minnesota Duluth, Department of Mathematics and Statistics, 2010.

7. Marshall Hall, Jr., Distinct representatives of subsets, Bull. Amer. Math. Soc. 54 (1948), 922-926.

8. Glenn Hurlbert, A survey of graph pebbling, Proceedings of the Thirtieth Southeastern International Conference on Combinatorics, Graph Theory, and Computing (Boca Raton, FL, 1999), vol. 139, 1999, pp. 41-64.

9. _ Recent progress in graph pebbling, Graph Theory Notes N. Y. 49 (2005), 25-37.

10. Brendan D. McKay, Practical graph isomorphism, Proceedings of the Tenth Manitoba Conference on Numerical Mathematics and Computing, Vol. I (Winnipeg, Man., 1980), vol. 30, 1981, pp. 45-87.

11. Jessica Muntz, Sivaram Narayan, Noah Streib, and Kelly Van Ochten, Optimal pebbling of graphs, Discrete Math. 307 (2007), no. 17-18, 2315-2321.

12. Lior Pachter, Hunter S. Snevily, and Bill Voxman, On pebbling graphs, Proceedings of the Twenty-sixth Southeastern International Conference on Combinatorics, Graph Theory and Computing (Boca Raton, FL, 1995), vol. 107, 1995, pp. 65-80.

13. László Papp, Optimal rubbling numbers of graphs (in Hungarian), thesis, Budapest Univewrsity of Technologoy and Economics, Department of Computer Science and Information Theory, 2010.

14. Nándor Sieben, A graph pebbling algorithm on weighted graphs, J. Graph Algorithms Appl. 14 (2010), no. 2, 221-244.

Budapest University of Technology and Economics Faculty of Electrical Engineering and Informatics, Department of Computer Science and Information Theory, H-1521 Budapest Po. BOX. 91, HuNGARY

E-mail address: kiskat@cs.bme.hu 
Northern Arizona University, Department of Mathematics and Statistics, Flagstaff AZ $86011-$ 5717, USA

E-mail address: nandor.sieben@nau.edu 